\title{
Nearly Complete Characterization of 2-Agent Deterministic Strategyproof Mechanisms for Single Facility Location in $L_{p}$ Space *
}

\author{
Jianan Lin \\ School of Computer Science, Fudan University, \\ Shanghai 201203, China \\ jnlin16@fudan.edu.cn
}

\begin{abstract}
We consider the problem of locating a single facility for 2 agents in $L_{p}$ space $(1<p<\infty)$ and give a nearly complete characterization of such deterministic strategyproof mechanisms. We use the distance between an agent and the facility in $L_{p}$ space to denote the cost of the agent. A mechanism is strategyproof iff no agent can reduce her cost from misreporting her private location.

We show that in $L_{p}$ space $(1<p<\infty)$ with 2 agents, any location output of a deterministic, unanimous, translation-invariant strategyproof mechanism must satisfy a set of equations and mechanisms are continuous, scalable. In one-dimensional space, the output must be one agent's location, which is easy to prove in any $n$ agents.

However, in $m$-dimensional space $(m \geq 2)$, the situation will be much more complex, with only 2 -agent case finished. We show that the output of such a mechanism must satisfy a set of equations, and when $p=$ 2 the output must locate at a sphere with the segment between the two agents as the diameter. Further more, for $n$-agent situations, we find that the simple extension of this the 2-agent situation cannot hold when dimension $m>2$ and prove that the well-known general median mechanism will give an counter-example.

Particularly, in $L_{2}$ (i.e., Euclidean) space with 2 agents, such a mechanism is rotation-invariant iff it is dictatorial; and such a mechanism is anonymous iff it is one of the three mechanisms in Section 4. And our tool implies that any such a mechanism has a tight lower bound of 2-approximation for maximum cost in any multi-dimensional space.
\end{abstract}

Keywords: Facility Location $\cdot$ Mechanism Design $\cdot L_{p}$ Space.

\section{Introduction}

We consider the problem of locating a single facility for $n$ (mainly in $n=2$ ) agents in $L_{p}$ space $(1<p<\infty)$. This facility serves these agents and every agent has a cost which is equal to the distance to access the facility. An agent's location is private information, i.e., only she herself knows it. A strategyproof

\footnotetext{
* Thanks for my advisors Pinyan Lu and $\mathrm{Hu}$ Fu for giving me advise on this problem.
} 
mechanism means that no agent can gain (i.e., reduce her cost) from misreporting her location. A mechanism is deterministic if the output is a specific location. Compared to randomized mechanisms, deterministic mechanisms often receive more attention because of their simplicity and ease of use.

A basic area of facility location study is the characterization of truthful mechanisms. In many situations and settings, the goal is to design a strategyproof mechanism which can minimize the objective cost function (e.g., social cost or maximum cost) as far as possible. Therefore, giving the characterization of such mechanisms will be helpful to further study. In this area, an important work is made by Moulin [10] that in any one-dimension space (they call it single-peaked preferences), every strategyproof, efficient (the selected alternative is Pareto optimal, which is different from our setting) and anonymous voting scheme (mechanism) must be a median voter scheme (to select the median agent). After that, Border and Jordan [4] extend his result to Euclidean space and show that it induces to median voter schemes in each dimension separately. Other works include Barberà et al. 22 that the result also fits in any $L_{1}$ norm, and [3] that in a compact set of the Euclidean space, which is a more restricted domain, all those mechanisms behave like generalized median voter schemes. Nearly all the relevant works focus on deterministic mechanisms and leaves the randomized ones an open question.

As for other settings, Tang et al. [13] firstly discuss the characterization of group-strategyproof (No group of agents can reduce their cost together by misreporting their location) both in deterministic and randomized mechanisms (The former characterization is complete and the latter is nearly complete). And Feigenbaum et al. 5] discuss the characterization of 2-agent randomized strategyproof mechanism in one-dimensional space. However, before our work, there has not been any discussion of the characterization of deterministic strategyproof mechanisms in any metric space.

One measurement of the facility location mechanisms is the cost they achieve. There are two common view: maximum cost (i.e., the maximum cost between the facility and some agent) and social cost (i.e., the sum of the cost between the facility and all the agents). The ratio between the cost one mechanism achieve and the minimum cost is widely used in this study. Procaccia and Tennenholtz 11] study approximately optimal strategyproof mechanisms for facility games both in maximum cost and social cost view, focusing on one-dimensional space and randomized mechanisms. They propose an interesting randomized mechanism for the maximum cost view, which achieves a ratio of $3 / 2$ and they proves it to be the best. Subsequently, Alon et al. 1] study the characterization of deterministic and randomized mechanisms in more general metric space (such as network and rings).

Other related works about facility location are $k$-facility location problems. Compared to single facility location problems, they are more complex. Agents can have preference on different facilities and their location can be public information this time. One important and classical work is made by Fotakis and Tzamos [8]. They study mechanisms that are winner-imposing, in the sense that 
the mechanisms allocate facilities to agents and require that each agent allocated a facility should connect to it. Also they prove an upper bound of $4 k$ in the social cost view. And there are many other follow-up works (see e.g., [7/12 6]14|9] ).

Our work is motivated by $[5$. Their result about the characterization of 2 agent randomized strategyproof mechanisms in a line (i.e., one-dimension) leads us studying the deterministic ones. And some of our technique is motivated by [13] (e.g., the proof of continuity). We show that in any one-dimensional $L_{p}$ space with $n$ agents, the output of a deterministic unanimous translation-invariant strategyproof mechanism should locate at one agent's location and in multidimensional $L_{p}$ space $(1<p<\infty)$ with 2 agents, the output of such a mechanism should satisfy a set of equations. Particularly, in $L_{2}$ space (i.e., the Euclidean space), let the two agents be $A, B$ and the output be $W$, then they should satisfy $\overrightarrow{W A} \cdot \overrightarrow{W B}=0$. These characterizations are nearly complete and next we give complete characterization of two more specific situations (also restricted in 2 agents). The first one is that such a mechanism is rotation-invariant if and only if it is dictatorial (i.e., the output location is always the same agent). The second one is that such a mechanism is anonymous (i.e., all the permutations of agents does not affect the output) if and only if it is one of the three mechanisms we give in Section 4. In the end, we show that the general median mechanism is an counter-example of the simple extension from 2-agent situation to $n$-agent situation in $m$-dimensional space $(m>2)$, which means that the characterization of $n$-agent situation may be very complex, unfortunately. Also, using our tool, we ensure a tight lower bound of 2-approximation for maximum cost in any multi-dimensional space.

\section{Preliminaries}

We consider the single facility location game with $n(n \geq 2)$ agents $N=$ $\{1,2, \ldots, n\}$. All the agents are located in a $m$-dimensional $L_{p}$ space $\mathbb{R}_{m}$. Obviously for $\forall x, y \in \mathbb{R}_{m}$, there is $\|x\|+\|y\| \geq\|x+y\|$ and the equality holds if and only if $x$ and $y$ have the same directions. We use $A_{i} \in \mathbb{R}_{m}$ to denote agent $i$ 's location in the space. Therefore a location profile is a vector consisting of all the agents' locations $\boldsymbol{A}=\left(A_{1}, A_{2}, \ldots, A_{n}\right)$.

A deterministic mechanism is a map $f: \mathbb{R}_{m}^{n} \rightarrow \mathbb{R}_{m}$ from a location profile to the location of the facility. We use $W=f(\boldsymbol{A})$ to denote the output location and the cost of agent $i$ is the distance between her and the facility, i.e., $d\left(A_{i}, W\right)=$ $\left\|W-A_{i}\right\|$. We will mix the two representations in this paper.

Next we formally define some properties of a deterministic mechanism.

Definition 1. (Strategyproofness). A mechanism $f$ is strategyproof if and only if no agent can reduce her distance to the output by misreporting her location. It means that, for $\forall \boldsymbol{A} \in \mathbb{R}_{m}^{n}, \forall i \in N, \forall A_{i}^{\prime} \in \mathbb{R}_{m}$, there is

$$
d\left(f(\boldsymbol{A}), A_{i}\right) \leq d\left(f\left(A_{i}^{\prime}, \boldsymbol{A}_{-i}\right), A_{i}\right) .
$$

Here $\boldsymbol{A}_{-i}=\left(A_{1}, \ldots, A_{i-1}, A_{i+1}, \ldots, A_{n}\right)$, i.e., the profile without $A_{i}$. 
Definition 2. (Unanimity). A mechanism $f$ is unanimous if and only if when $\forall A_{i}=C$, we have

$$
f(\boldsymbol{A})=C,
$$

which means that if all agents report the same location, then the mechanism must output this location.

Definition 3. (Dictatorship). A mechanism $f$ is dictatorial if and only if $\exists i \in$ $N, \forall A \in \mathbb{R}_{m}^{n}$, there is

$$
f(\boldsymbol{A})=A_{i} .
$$

At this time we call $i$ is the dictator.

Definition 4. (Anonymity). A mechanism $f$ is anonymous if and only if when any group of the agents exchange their location reports, the output is still the same, which means that any permutation of the agents' locations does not affect the output.

Definition 5. (Translational Invariance). A mechanism $f$ is translation-invariant if and only if

$$
\forall \boldsymbol{A} \in \mathbb{R}_{m}^{n}, \forall t \in \mathbb{R}_{m}, f(\boldsymbol{A}+t)=f(\boldsymbol{A})+t .
$$

Here, $f(\boldsymbol{A}+t)=f\left(A_{1}+t, \ldots, A_{n}+t\right)$. This means that if we move all the agents the same distance in one direction, then the output location will also move the same distance in this direction.

Definition 6. (Scalability). A mechanism $f$ is scalable if and only if

$$
\forall \boldsymbol{A} \in \mathbb{R}_{m}^{n}, \forall k \in \mathbb{R}, k>0, f(k \cdot \boldsymbol{A})=k \cdot f(\boldsymbol{A}) .
$$

Here, $f(k \cdot \boldsymbol{A})=f\left(k \cdot A_{1}, \ldots, k \cdot A_{n}\right)$.

Notice that if a mechanism $f$ satisfies translational invariance and scalability, then we will have

$$
\forall \boldsymbol{A} \in \mathbb{R}_{m}^{n}, \forall k \in \mathbb{R}, k>0, \forall t \in \mathbb{R}_{m}, f(k \cdot \boldsymbol{A}+t)=k \cdot f(\boldsymbol{A})+t .
$$

Definition 7. (Rotational Invariance). A mechanism $f$ is rotation-invariant if and only if when all the agents are rotated at the same angle around a point (not necessary to be an agent) in the same direction in some dimensions, then the output will also be rotated at this angle around the point in such direction in these dimensions.

For convenience, the properties of rotational invariance and anonymity are only described with natural languages. Notice that the description of rotational invariance includes situations that points are rotated on an axis and so on, because of "in some dimensions". 


\section{Nearly Complete Characterization of Deterministic Mechanisms}

We will start with the situations in one-dimensional space as a warm-up and prove that this characterization is suitable for any $n$ agents. Then we will discuss the multi-dimensional situations in $L_{p}$ space with 2 agents for $1<p<\infty$. The reason why we abandon $L_{1}$ and $L_{\infty}$ is that in these two spaces, there exists two vectors $x, y$ with different directions that $\|x\|+\|y\|=\|x+y\|$, which is not a friendly property. We use $m$ to denote number of dimensions.

\subsection{One-Dimensional Situation}

The one-dimensional situation is simple. In any $L_{p}$ space, $\forall a, b, c \in \mathbb{R}$, if $a \leq b \leq$ $c$, then we have $\|a-b\|+\|b-c\|=\|a-c\|$. For convenience, we call the negative direction in the coordinate axis "left" and call the positive direction "right".

Lemma 1. (Continuity) If mechanism $f$ is strategyproof, then for $\forall i \in N$ with any fixed $\boldsymbol{A}_{-i} \in \mathbb{R}_{m}^{n-1}$, we have

$$
\left\|u\left(A_{i}\right)-u\left(A_{i}^{\prime}\right)\right\| \leq\left\|A_{i}-A_{i}^{\prime}\right\|
$$

where $u\left(A_{i}\right)=\left\|f\left(A_{i}, \boldsymbol{A}_{-i}\right)-A_{i}\right\|$. This implies that $u\left(A_{i}\right)$ is a continuous function.

Proof. We assume that $\exists A_{i}, A_{i}^{\prime}$ such that $\left\|u\left(A_{i}\right)-u\left(A_{i}^{\prime}\right)\right\|>\left\|A_{i}-A_{i}^{\prime}\right\|$. Also without loss of generality, we assume that $u\left(A_{i}\right)>u\left(A_{i}^{\prime}\right)$ which means that $u\left(A_{i}\right)-u\left(A_{i}^{\prime}\right)>\left\|A_{i}-A_{i}^{\prime}\right\|$, then we have

$$
\begin{aligned}
\left\|f\left(A_{i}^{\prime}, \boldsymbol{A}_{-i}\right)-A_{i}\right\| & \leq\left\|f\left(A_{i}^{\prime}, \boldsymbol{A}_{-i}\right)-A_{i}^{\prime}\right\|+\left\|A_{i}-A_{i}^{\prime}\right\| \\
& =u\left(A_{i}^{\prime}\right)+\left\|A_{i}-A_{i}^{\prime}\right\| \\
& <u\left(A_{i}\right)=\left\|f\left(A_{i}, \boldsymbol{A}_{-i}\right)-A_{i}\right\| .
\end{aligned}
$$

Notice this is contradict with the strategyproofness, because $A_{i}$ can misreport her location as $A_{i}^{\prime}$ to reduce her cost. Therefore the previous inequality in lemma must hold. When $A_{i}^{\prime} \rightarrow A_{i}$, we see $u\left(A_{i}\right)$ is a continuous function.

This lemma is very useful and fits any $m$ (dimensions) and $n$ (agents).

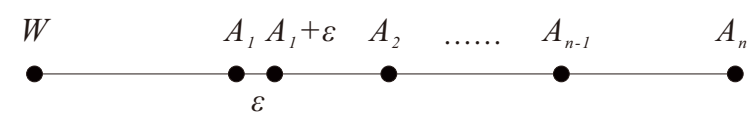

Fig. 1. Case 1 in the proof of Theorem 1

Theorem 1. When $m=1$, the output of a deterministic unanimous translationinvariant strategyproof mechanism must be one agent's location. 
Proof. Without loss of generality, we let $n$ agents be $A_{1}, \ldots, A_{n}$ and assume $A_{1} \leq A_{2} \leq \ldots \leq A_{n}$. If all $A_{i}$ are in the same location, according to unanimity, the output is $A_{i}$.

Let $W=f(\boldsymbol{A})$. We divide this into 3 different cases. We only need to prove that the output cannot locate at these three areas. Using proof by contradiction, we assume that there can be a situation that $W$ does not locate at any agents.

Case 1, $W<A_{1}$ : As is shown in Fig 1, we can find a positive tiny $\epsilon$ that $\epsilon \ll d\left(W, A_{1}\right)$, e.g., $\epsilon<0.01 \cdot d\left(W, A_{1}\right)$ and according to Lemma 1 , we have $f\left(A_{1}+\epsilon, \boldsymbol{A}_{-\mathbf{1}}\right)<A_{1}$, otherwise it will contradict with $\left\|u\left(A_{1}\right)-u\left(A_{1}+\epsilon\right)\right\| \leq$ $\mid A_{1}-\left(A_{1}+\epsilon\right) \|=\epsilon$.

Therefore we must have $f\left(A_{1}+\epsilon, \boldsymbol{A}_{-1}\right) \leq W$, otherwise agent with location $A_{1}$ gain from misreporting her location as $A_{1}+\epsilon$. In the same way, we also must have $f\left(A_{1}+\epsilon, \boldsymbol{A}_{-1}\right) \geq W$, otherwise agent with location $A_{1}+\epsilon$ can gain from misreporting her location as $A_{1}$ (fix other agents in $\boldsymbol{A}_{-\mathbf{1}}$ ). This means that $f\left(A_{1}+\epsilon, \boldsymbol{A}_{-1}\right)=f(\boldsymbol{A})=W$.

Let $\boldsymbol{A}_{-i}^{\epsilon}$ denotes $\left(A_{1}+\epsilon, \ldots, A_{i-1}+\epsilon, A_{i+1}, \ldots, A_{n}\right)$ (of course $1 \leq i \leq n$ and when $i=n$ we say it denotes $\left(A_{1}+\epsilon, \ldots, A_{n-1}+\epsilon\right.$, when $i=1$ we say it denotes $\left(A_{2}, \ldots, A_{n}\right)$. In the same way, when $i$ increases from 1 to $n$, we have $f\left(A_{n}+\epsilon, \boldsymbol{A}_{-\boldsymbol{n}}^{\boldsymbol{\epsilon}}\right)=f\left(A_{n-1}+\epsilon, \boldsymbol{A}_{-(\boldsymbol{n}-\mathbf{1})}^{\boldsymbol{\epsilon}}\right)=\ldots=f(\boldsymbol{A})=W$ and at last get $f(\boldsymbol{A}+\epsilon)=W$. However, according to the translational-invariance, we must have $f(\boldsymbol{A})=W+\epsilon$, which leads to a contradiction. Therefore the output cannot satisfy $W<A_{1}$.

Case 2, $W>A_{n}$ : This is completely symmetrical with the first case and we can use the same method by adding a tiny $\epsilon\left(\epsilon \ll d\left(W, A_{n}\right)\right)$ to all agents.

Case 3, $\exists i \in[1, n-1], A_{i}<W<A_{i+1}$ : We can still add all the agents a tiny $\epsilon \ll \min \left\{d\left(W, A_{i}\right), d\left(W, A_{i+1}\right)\right\}$. When adding $A_{j}$ with $j \leq i$, we can refer to case 1's proof and when adding $A_{j}$ with $j>i$, we can refer to case 2's method.

Notice that if $A_{i}=A_{i+1}$, then there cannot be $A_{i}<W<A_{i+1}$, thus these 3 cases include all the areas except the locations of the agents. Therefore, any such strategyproof mechanism cannot output a location $W \neq A_{i}$ for any $i$. We prove this theorem.

Therefore we can know that in one-dimensional space (including all the $L_{p}$ space for any positive integer $p$ ), the output must be one agent's location. But in multi-dimensional space, the result is different. Although output still can be one agent's location, it's not necessary.

\subsection{Multi-Dimensional $L_{2}$ Situation}

There are two reasons why we select $L_{2}$ space (i.e., Euclidean space). The first one is that it has some very friendly properties. For example, in an Euclidean space, any right triangle must satisfy that its hypotenuse is the (only) longest side. But in other space such as $L_{3}$ space, this rule may not hold. Here, the length of side is the distance between the two points in $L_{p}$ space. And the second reason is that the Euclidean space is the most common and most used space. In this part, we will study the result in Euclidean space. 
Lemma 2. In any Euclidean space with $n$ agents $A_{i}(i \in N)$, let the output of a deterministic unanimous translation-invariant strategyproof mechanism be $W$, then for $\forall A_{i}^{\prime}$ on the segment between $A_{i}$ and $W$ (including $A_{i}$ and $W$ ), we have

$$
f\left(A_{i}^{\prime}, \boldsymbol{A}_{-i}\right)=W
$$

which means that if one agent move her location close to the output along the segment, the output does not change.

Proof. Obviously we only need to care the situation that $A_{i}^{\prime} \neq A_{i}$. Considering the property of strategyptoofness and let $W^{\prime}=f\left(A_{i}^{\prime}, \boldsymbol{A}_{-i}\right)$, we have

$$
\left\{\begin{array}{l}
d\left(A_{i}, W\right) \leq d\left(A_{i}, W^{\prime}\right) \\
d\left(A_{i}^{\prime}, W^{\prime}\right) \leq d\left(A_{i}^{\prime}, W\right)
\end{array}\right.
$$

Draw the spheres (if $m=2$ then circles and if $m>3$ then $m$-spheres) $O_{1}$ and $O_{2}$ with $A_{i}$ and $A_{i}^{\prime}$ as centers, $d\left(A_{i}, W\right)$ and $d\left(A_{i}^{\prime}, W\right)$ as radius, respectively. The first inequality implies that $W^{\prime}$ cannot be inside of $O_{1}$ and the second implies that $W^{\prime}$ cannot be outside of $O_{2}$. Therefore, $W^{\prime}=W$, which is the only common point between the two spheres (circles).

In fact, this lemma holds when $p>2$, but at this time, what we draw is not 2 spheres any more, but 2 inscribed similar Enclosed ellipsoid on which the distance between a point and center is a constant in $L_{p}$ space.

Lemma 3. In any Euclidean space with 2 agents $A$ and $B$, if the output of a deterministic unanimous translation-invariant strategyproof mechanism is on the line $A B$, then it can only locate at $A$ or $B$.

Proof. Similar to the proof of Theorem 1, we set the output $W$ and divide it into three cases.

If $\exists k>0$ that $\overrightarrow{W A}=k \cdot \overrightarrow{A B}$, then according to lemma 2, we move $A$ and then $B$ towards $W$ with a tiny distance $\epsilon$. In this period, the output is still $W$, which is contradict with translational-invariance.

If $\exists k>0$ that $\overrightarrow{W B}=k \cdot \overrightarrow{B A}$, then according to lemma 2, we move $B$ and then $A$ towards $W$ with a tiny distance $\epsilon$. In this period, the output is still $W$, which is contradict with translational-invariance.

If $\exists k>0$ that $\overrightarrow{A W}=k \cdot \overrightarrow{W B}$, then according to lemma 2, we move $A$ to $W$ and have $f(W, B)=W$. Next we move $B$ away from $W$ to $B^{\prime}$ so that $d\left(B^{\prime}, W\right)=d(A, B)$. Therefore we notice that $f\left(W, B^{\prime}\right) \neq W+(W-A)$ because otherwise $B$ can gain from misreporting $B^{\prime}$, and this is contradict with translational-invariance.

Therefore, the lemma is proved.

Lemma 4. In any Euclidean space with 2 agents $A$ and $B$, the output $W$ can never satisfy that $0^{\circ}<\angle A W B<90^{\circ}$. 


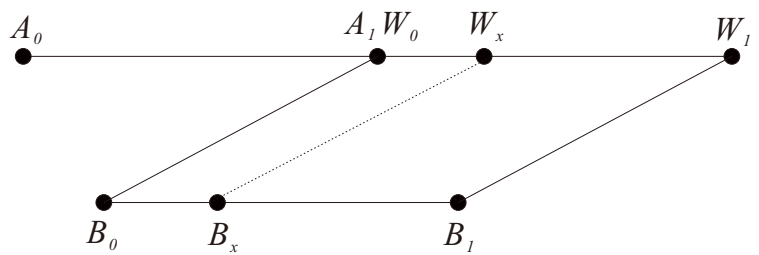

Fig. 2. Proof of Lemma 4

Proof. For convenience, we use term $A_{x}$ and $B_{x}(x \in[0,1])$ and $W_{x}=f\left(A_{x}, B_{x}\right)$. Let $\angle A_{0} W_{0} B_{0} \in\left(0^{\circ}, 90^{\circ}\right)$. Also let $\overrightarrow{B_{0} B_{1}}=\overrightarrow{A_{0} W_{0}}$ and $A_{1}=W_{0}$. Therefore we have $\overrightarrow{W_{0} W_{1}}=\overrightarrow{B_{0} B_{1}}$. Here $\overrightarrow{A_{0} A_{x}}=x \cdot \overrightarrow{A_{0} A_{1}}, \overrightarrow{B_{0} B_{x}}=x \cdot \overrightarrow{B_{0} B_{1}}$ and $\overrightarrow{W_{0} W_{x}}=$ $x \cdot \overrightarrow{W_{0} W_{1}}$. These are all drawn in Fig 2 .

According to Lemma 2, because $f\left(A_{x}, B_{x}\right)=W_{x}$, we can get $f\left(A_{1}, B_{x}\right)=$ $W_{x}$. This means that if we fix $A$ in $A_{1}$ and move $B$ from $B_{0}$ to $B_{1}$ on a straight line, then $W$ will move from $W_{0}$ to $W_{1}$ on a straight line. Because of strategyproofness, for $\forall x \in[0,1]$, we have $d\left(B_{1}, W_{x}\right) \geq d\left(B_{1}, W_{1}\right)$. However, since we know that $\angle A_{1} W_{1} B_{1}=\angle A_{0} W_{0} B_{0}<90^{\circ}$, then there must exist $x<1$ so that $d\left(B_{1}, W_{x}\right)<d\left(B_{1}, W_{1}\right)$. Therefore the lemma is proved.

Lemma 5. In any Euclidean space with 2 agents $A$ and $B$, the output $W$ can never satisfy that $90^{\circ}<\angle A W B<180^{\circ}$.

Proof. Similar to the proof of Lemma 4, we use term $A_{x}$ and $B_{x}(x \in[0,1])$ and $W_{x}=f\left(A_{x}, B_{x}\right)$. Let $\angle A_{0} W_{0} B_{0} \in\left(90^{\circ}, 180^{\circ}\right)$. Also let $\overrightarrow{B_{0} B_{1}}=\overrightarrow{A_{0} W_{0}}$ and $A_{1}=W_{0}$. Therefore we have $\overrightarrow{W_{0} W_{1}}=\overrightarrow{B_{0} B_{1}}$. Here $\overrightarrow{A_{0} A_{x}}=x \cdot \overrightarrow{A_{0} A_{1}}$, $\overrightarrow{B_{0} B_{x}}=x \cdot \overrightarrow{B_{0} B_{1}}$ and $\overrightarrow{W_{0} W_{x}}=x \cdot \overrightarrow{W_{0} W_{1}}$.

According to Lemma 2, because $f\left(A_{x}, B_{x}\right)=W_{x}$, we can get $f\left(A_{1}, B_{x}\right)=$ $W_{x}$. This means that if we fix $A$ in $A_{1}$ and move $B$ from $B_{0}$ to $B_{1}$ on a straight line, then $W$ will move from $W_{0}$ to $W_{1}$ on a straight line. Because of strategyproofness, for $\forall x \in[0,1]$, we have $d\left(B_{0}, W_{x}\right) \geq d\left(B_{0}, W_{0}\right)$. However, since we know that $\angle W_{1} A_{1} B_{0}<90^{\circ}$, then there must exist $x>0$ so that $d\left(B_{0}, W_{x}\right)<d\left(B_{0}, W_{0}\right)$. Therefore the lemma is proved.

Theorem 2. In any Euclidean space with 2 agents $A$ and $B$, the output $W$ of a deterministic unanimous translation-invariant strategyproof mechanism $f$ must satisfy

$$
\overrightarrow{A W} \cdot \overrightarrow{B W}=0
$$

which means that $W$ must locate on a sphere with $A B$ as the diameter.

According to Lemma 3, 4 and 5, Theorem 2 is obvious. Notice this theorem fits any dimension $m>1$. Maybe intuitively we can guess that this can be extended to more $n$-agent situation, but we will show that there is an counterexample for any $m>2$ in Section 5. Here is the conjecture that does not hold for $m>2$.

Conjecture 1. In any Euclidean space with $n$ agents $A_{1}, \ldots, A_{n}$, the output $W$ of a deterministic unanimous translation-invariant strategyproof mechanism $f$ must satisfy that

$$
\exists i, j \in N, \overrightarrow{A_{i} W} \cdot \overrightarrow{A_{j} W}=0 .
$$




\subsection{Multi-Dimensional $L_{p}$ Situation}

As is mentioned in the last part, we know that other $L_{p}$ space has less friendly properties than $L_{2}$ space. Therefore the result is not a right angle any more because in other $L_{p}$ space, a right triangle's hypotenuse may not be the longest side. We use analytical geometry to solve this problem. Obviously we only need to analyze the case that the output $W$ does not locate at $A$ or $B$.

According to translational-invariance, We can assume that $A\left(-x_{1}, \ldots,-x_{m}\right)$, $B\left(x_{1}, \ldots, x_{m}\right)$ and $W\left(y_{1}, \ldots, y_{m}\right)$, then the distance between two points such as $A, W$ is $d_{p}(A, W)=\left(\sum_{i=1}^{m}\left|x_{i}+y_{i}\right|^{p}\right)^{1 / p}$.

Theorem 3. In any $L_{p}$ space $(2<p<\infty)$ with 2 agents $A$ and $B$, the output $W$ of a deterministic unanimous translation-invariant strategyproof mechanism $f$ must satisfy

$$
\left\{\begin{array}{l}
\sum_{i=1}^{m}\left(x_{i}+y_{i}\right) \cdot\left(x_{i}-y_{i}\right) \cdot\left|x_{i}-y_{i}\right|^{p-2}=0 \\
\sum_{i=1}^{m}\left(x_{i}-y_{i}\right) \cdot\left(x_{i}+y_{i}\right) \cdot\left|x_{i}+y_{i}\right|^{p-2}=0
\end{array}\right.
$$

Proof. Consider such a situation. In a $L_{p}$ space, let $f\left(A_{0}, B_{0}\right)=W_{0}, A_{1}=$ $W_{0}$ and $\overrightarrow{B_{0} B_{1}}=\overrightarrow{A_{0} A_{1}}$, therefore we have $\overrightarrow{W_{0} W_{1}}=\overrightarrow{B_{0} B_{1}}$. We still use term $A_{x}, B_{x}, W_{x}$ which mean $\overrightarrow{A_{0} A_{x}}=\overrightarrow{A_{0} A_{1}}, \overrightarrow{B_{0} B_{x}}=\overrightarrow{B_{0} B_{1}}$ and $\overrightarrow{W_{0} W_{x}}=\overrightarrow{W_{0} W_{1}}$ respectively.

Similar to the proof of Lemma 4 and Lemma 5, we can easily find that $f\left(A_{1}, B_{x}\right)=W_{x}$. Considering strategyproofness, because we know that when $B$ moves from $B_{0}$ to $B_{1}$ with fixed $A_{1}, W_{x}$ moves from $W_{0}$ to $W_{1}$, then $d\left(B_{x}, W_{x}\right)$ is the shortest distance between $B_{x}$ and segment $\overline{W_{0} W_{1}}$, otherwise agent $B$ at $B_{x}$ may misreport her location $B_{x}^{\prime}$ to reduce her cost.

Because of translational-invariance, let $\alpha \in[-1,1]$. Using $A, B, W$ instead of $A_{0}, B_{0}, W_{0}$, we set

$$
g(\alpha)=\left(\sum_{i=1}^{m}\left|\left(y_{i}+\alpha \cdot\left(y_{i}+x_{i}\right)-x_{i}\right)\right|^{p}\right)^{1 / p} .
$$

Obviously, $g(\alpha)$ means the distance between $B$ and some point in segment $\overline{A W}$. According to the last paragraph, we have $g(0)=\min _{\alpha \in[-1,1]} g(\alpha)$, and $g^{\prime}(0)=0$ which means derivative of $g(\alpha)$.

In the same way, let $h(\beta)(\beta \in[-1,1])$ denotes the distance between $A$ and some point in segment $\overline{B W}$ and we will have

$$
h(\beta)=\left(\sum_{i=1}^{m}\left|y_{i}+\beta \cdot\left(y_{i}-x_{i}\right)+x_{i}\right|^{p}\right)^{1 / p},
$$

and $h^{\prime}(0)=0$. 
For convenience, let $G(\alpha)=g(\alpha)^{p} / p$ and $H(\alpha)=h(\alpha)^{p} / p$, thus $G^{\prime}(0)=$ $H^{\prime}(0)=0$. We have

$$
\left\{\begin{array}{l}
G^{\prime}(\alpha)=\sum_{i=1}^{m}\left(y_{i}+\alpha \cdot\left(x_{i}+y_{i}\right)-x_{i}\right) \cdot\left|y_{i}+\alpha \cdot\left(x_{i}+y_{i}\right)-x_{i}\right|^{p-2} \cdot\left(x_{i}+y_{i}\right) \\
H^{\prime}(\beta)=\sum_{i=1}^{m}\left(y_{i}+\beta \cdot\left(y_{i}-x_{i}\right)+x_{i}\right) \cdot\left|y_{i}+\beta \cdot\left(y_{i}-x_{i}\right)+x_{i}\right|^{p-2} \cdot\left(y_{i}-x_{i}\right)
\end{array}\right.
$$

Considering $G^{\prime}(0)=H^{\prime}(0)=0$, we have

$$
\left\{\begin{array}{l}
\sum_{i=1}^{m}\left(x_{i}+y_{i}\right) \cdot\left(x_{i}-y_{i}\right) \cdot\left|x_{i}-y_{i}\right|^{p-2}=0 \\
\sum_{i=1}^{m}\left(x_{i}-y_{i}\right) \cdot\left(x_{i}+y_{i}\right) \cdot\left|x_{i}+y_{i}\right|^{p-2}=0
\end{array}\right.
$$

In summary, the equations in the theorem hold.

We can find that the group of equations has an infinite number of solutions if and only if $p=2$ (When $p=2,\left|x_{i} \pm y_{i}\right|^{p-2}$ in the equations should be replaced with 1 ). And at this time the two equations are equivalent which mean the output should locate on a sphere with $A B$ as the diameter.

Theorem 4. In any $L_{p}$ space $(1<p<\infty)$ with 2 agents $A$ and $B$, a deterministic unanimous translation-invariant strategyproof mechanism $f$ must be scalable.

Proof. When $p>2$, according to Theorem 3, because of finite number of valid output locations, the property scalability holds, otherwise it will contradict with continuity and translational-invariance (Let's imagine a situation: We move one agent to the other slowly, and if the output does not obey scalability, then it will "jump" in some time to another valid output location). Therefore we only need to discuss $p=2$.

Considering translational-invariance, we can assume $A$ locates at the origin. then we have $f(k \cdot A, k \cdot B)=f(A, k \cdot B)$, which means we only need to move $B$. Notice that for any $k_{1} \cdot k_{2}=1$, we find $f\left(k_{1} \cdot A, k_{1} \cdot B\right)$ and $f\left(k_{2} \cdot A, k_{2} \cdot B\right)$ are inverted to each other. Therefore we only need to analyze $0<k<1$ (Of course we do not need to discuss when $k=1)$. Then we divide this into 3 cases.

Case $1, f(A, B)=A$ : According to Lemma $2, \forall k \in(0,1)$, we have $f(A, k$. $B)=A$.

Case 2, $f(A, B)=B$ : According to Lemma 2, $\forall k \in(0,1)$, we have $f(A+$ $(1-k) \cdot B, B)=B$. Considering translational-invariance, we have $f(A, k \cdot B)=$ $f(A+(1-k) \cdot B-(1-k) \cdot B, B-(1-k) \cdot B)=B-(1-k) \cdot B=k \cdot B$.

Case 3, $f(A, B) \neq A, B$ : Assume $W=f(A, B), B^{\prime}=k \cdot B$, and $W^{\prime}=$ $f\left(A, B^{\prime}\right)$. Therefore, there exists $C \in \overline{A W}$ and $D \in \overline{B W}$ that $\overrightarrow{C D}=\overrightarrow{A B^{\prime}}=$ 
$k \cdot \overrightarrow{A B}$. Obviously $\triangle W C D \cong \triangle W^{\prime} A B^{\prime}$. Thus we know $W^{\prime} \in \overrightarrow{A W}, \overrightarrow{A W^{\prime}}=k \cdot \overrightarrow{A W}$ and $\overrightarrow{W^{\prime} B^{\prime}}=\overrightarrow{W B}$. This means that $W^{\prime}=k \cdot W$.

Also, we give our conjecture about the $n$-agent situation.

Conjecture 2. In any $L_{p}(1<p<\infty)$ space with $n$ agents, a deterministic unanimous translation-invariant strategyproof mechanism $f$ is scalable.

\section{Two Special Cases}

Although we cannot give complete characterization of any 2-agent deterministic unanimous translation-invariant strategyproof mechanism, we finish two special cases. One is dictatorial mechanism in Euclidean spcae, and the other is anonymous mechanism in 2-dimensional Euclidean space.

\subsection{Dictatorial Mechanisms}

Theorem 5. In any Euclidean space with 2 agents, $f$ is a deterministic unanimous translation-invariant strategyproof mechanism, then $f$ is rotation-invariant if and only if $f$ is dictatorial.

Proof. First of all, if $f$ is dictatorial, then obviously it is rotation-invariant. Then we only need to analyze the case that $f$ is rotation-invariant.

Let 2 agents be $A$ and $B$, and we assume $W=f(A, B)$. If there exists $A_{0}, B_{0}$ that $W_{0}=f\left(A_{0}, B_{0}\right) \neq A_{0}, B_{0}$, then we have $f\left(W_{0}, B_{0}\right)=W_{0}$. Otherwise we can imagine an agent $A$ with location $W_{0}$ misreports her location as $A_{0}$ to reduce her cost. Besides, we know that $f$ is scalable and translation-invariant, then if we move $B_{0}$ to some $B_{1} \in \overline{A_{0} B_{0}}$ so that $\left\|A_{0} B_{1}\right\|=\left\|W_{0} B_{0}\right\|$. Therefore we will find that it is contradict with rotational-invariance by observing $f\left(A_{0}, B_{1}\right)$ and $f\left(W_{0}, B_{0}\right)$. Thus the output can only locate at $A$ or $B$.

Consider the following three properties: rotational-invariance, translationalinvariance and scalability, and we will find that if $f\left(A_{0}, B_{0}\right)=A_{0}$ (or $B_{0}, A_{0} \neq$ $B_{0}$ otherwise we can solve this by unanimity), then for $\forall A, B \in \mathbb{R}_{m}$, we have $f(A, B)=A$ (or $B$ ), because any two points in $L_{p}$ space can be transformed by $A_{0}$ and $B_{0}$ with these three properties. Therefore, $f$ is dictatorial.

\subsection{Anonymous Mechanisms}

Here we give 3 anonymous mechanisms in 2-dimensional Euclidean space. Let 2 agents be $A$ and $B$ with different coordinates $\left(x_{A}, y_{A}\right)$ and $\left(x_{B}, y_{B}\right)$ respectively. For these 3 mechanisms, if $A=B$, then we select $A$ (or $B$ ) as the facility.

Mechanism $1:(u, v)-\mathrm{C} 1 \operatorname{Mechanism}(u, v \in\{0,1\})$.

If $u=1$, then $x_{W}=\max \left\{x_{A}, x_{B}\right\}$; if $u=0$, then $x_{W}=\min \left\{x_{A}, x_{B}\right\}$.

If $v=1$, then $y_{W}=\max \left\{y_{A}, y_{B}\right\}$; if $v=0$, then $y_{W}=\min \left\{y_{A}, y_{B}\right\}$. 
Mechanism 2 : $(u)-\mathrm{C} 2$ Mechanism $(u \neq 0)$. Without loss of generality, we assume $x_{A} \leq x_{B}$. And $W=f(A, B)$ with coordinate $\left(x_{W}, y_{W}\right)$. We divide this into three cases.

Case 1. When $x_{A}=x_{B}$, we let $x_{W}=x_{A}$. If $u>0$, let $y_{W}=\max \left\{y_{A}, y_{B}\right\}$ and if $u<0$, let $y_{W}=\min \left\{y_{A}, y_{B}\right\}$.

Notice other 2 cases satisfy $x_{A}<x_{B}$. Let $R=\left(y_{B}-y_{A}\right) /\left(x_{B}-x_{A}\right)$.

Case 2. $u>0$. When $-1 / u \leq R \leq u$, draw line (1) $y=u \cdot\left(x-x_{A}\right)+y_{A}$ and line (2) $y=-\frac{1}{u} \cdot\left(x-x_{B}\right)+y_{B}$. Let $W$ be the intersection of the two lines. When $R>u$, we let $W=A$ and when $R<-1 / u$, we let $W=B$.

Case 3. $u<0$. When $u \leq R \leq-1 / u$, draw line (1) $y=u \cdot\left(x-x_{A}\right)+y_{A}$ and line (2) $y=-\frac{1}{u} \cdot\left(x-x_{B}\right)+y_{B}$. Let $W$ be the intersection of the two lines. When $R>-1 / u$, we let $W=A$ and when $R<u$, we let $W=B$.

Mechanism 3 : $(v)$-C3 Mechanism $(v \neq 0)$. Without loss of generality, we assume $y_{A} \leq y_{B}$. And $W=f(A, B)$ with coordinate $\left(x_{W}, y_{W}\right)$. We divide this into three cases.

Case 1. When $y_{A}=y_{B}$, we let $y_{W}=y_{A}$. If $v>0$, let $x_{W}=\max \left\{x_{A}, x_{B}\right\}$ and if $v<0$, let $x_{W}=\min \left\{x_{A}, x_{B}\right\}$.

Notice other 2 cases satisfy $x_{A}<x_{B}$. Let $S=\left(x_{B}-x_{A}\right) /\left(y_{B}-y_{A}\right)$.

Case 2. $v>0$. When $-1 / v \leq S \leq v$, draw line (1) $x=v \cdot\left(y-y_{A}\right)+x_{A}$ and line (2) $x=-\frac{1}{v} \cdot\left(y-y_{B}\right)+x_{B}$. Let $W$ be the intersection of the two lines. When $S>v$, we let $W=A$ and when $S<-1 / v$, we let $W=B$.

Case 3. $v<0$. When $v \leq S \leq-1 / v$, draw line (1) $x=v \cdot\left(y-y_{A}\right)+x_{A}$ and line (2) $x=-\frac{1}{v} \cdot\left(y-y_{B}\right)+x_{B}$. Let $W$ be the intersection of the two lines. When $S>-1 / v$, we let $W=A$ and when $S<v$, we let $W=B$.

Theorem 6. In any 2-dimensional Eucildean space with 2 agents, a mechanism $f$ is deterministic unanimous translation-invariant anonymous strategyproof, if and only if $f$ is one of Mechanism 1, 2, and 3.

Proof. Firstly we prove sufficiency. Obviously if $f$ is one of the 3 mechanisms, it is deterministic, unanimous, translation-invariant and anonymous. So we only need to prove it is strategyproof. Considering translational-invariance, anonymity and scalability (proved in previous theorem), we only need to prove for any $A_{0}(0,0)$ and $B_{0}(\cos \theta, \sin \theta), B$ cannot gain from misreporting her location.

Mechanism 1 is strategyproof: Out of symmetry, we only need to prove $(1,1)$ C1 Mechanism is strategyproof.

(1) When $\theta \in[0, \pi / 2], B$ will never misreport because her cost is 0 .

(2) When $\theta \in(\pi / 2, \pi)$, the facility is $(0, \sin \theta)$ and $B$ 's $\operatorname{cost}$ is $-\cos \theta$. Because $A_{0}(0,0)$, then $x_{W} \geq 0$, therefore this is the minimum cost for $B$.

(3) When $\theta \in[\pi, 3 \pi / 2]$, the facility is $(0,0)$ and $B$ 's cost is 1 . Because $A_{0}(0,0)$, then $x_{W} \geq 0$ and $y_{W} \geq 0$, therefore this is the minimum cost for $B$.

(4) When $\theta \in(3 \pi / 2,2 \pi)$, we can use the same way as (2) to prove.

Mechanism 2 and 3 are strategyproof: Out of symmetry, we only need to prove Mechanism 2 is strategyproof for $u>0$ and $\theta \in[-\pi / 2, \pi / 2]$ (And we fill find this time $x_{B} \geq x_{A}$ ).

(1) When $\theta \in[\arctan u-\pi / 2, \arctan u]$, then $W_{0} \in$ Line $1(y=u x)$ and $x_{W} \geq 0$. 
We assume $B$ misreports as $B^{\prime}\left(x^{\prime}, y^{\prime}\right)$. When $y^{\prime} \geq 0$, if $y^{\prime} / x^{\prime}>u$ or $\leq 0$ or $x^{\prime}=0$, then the output will locate at top left of Line 1, leading the cost larger than previous one; if $y^{\prime} / x^{\prime} \in[0, u]$, then the output will locate at Line 1, leading the cost never smaller than the previous one because $A_{0} W_{0} \perp B_{0} W_{0}$ and in Euclidean space this is the smallest distance. When $y^{\prime}<0$, in the same way, if $-1 / u<y^{\prime} / x^{\prime}<0$, the output will locate at Line 1 ; if $y^{\prime} / x^{\prime}<-1 / u$ or $>u$ or $x^{\prime}=0$, the output will locate at $A$; and if $0<y^{\prime} / x^{\prime}<u$, the output will locate at top left of Line 1 .

(2) When $\theta \in(\arctan u, \pi / 2], B$ will never misreport because $W_{0}=B_{0}$.

(3) When $\theta \in[-\pi / 2, \arctan u-\pi / 2)$, then $W_{0}=A_{0}$ and the cost is 1 . We assume $B$ misreports as $B^{\prime}\left(x^{\prime}, y^{\prime}\right)$. When $y^{\prime} \leq 0$, if $y^{\prime} / x^{\prime} \leq-1 / u$ or $\geq u$ or $x^{\prime}=0$, the output will still locate at $A_{0}$; if $-1 / u<y^{\prime} / x^{\prime} \leq 0$, the output $W^{\prime} \in$ Line 1 and $\angle B_{0} A_{0} W^{\prime}>90^{\circ}$ so the cost will increase; if $0<y^{\prime} / x^{\prime}<u$, the output $W^{\prime} \in$ Line 2 and $\angle B_{0} A_{0} W^{\prime}>90^{\circ}$ so the cost will increase. When $y^{\prime}>0$, in the same way, we have $\angle B_{0} A_{0} W^{\prime}>90^{\circ}$.

Secondly, we prove necessity. In fact, we only need to observe the output of $f((0,-1),(0,1))$ and $f((-1,0),(1,0))$ and this is enough for us to characterize the whole mechanism. According to Theorem 2, any output $W$ must satisfy $\overrightarrow{W A} \cdot \overrightarrow{W B}=0$. We divide this into 4 cases.

(1) $f((0,-1),(0,1))=(0, \pm 1)$ and $f((-1,0),(1,0))=( \pm 1,0)$. Out of symmetry, we mainly discuss the positive result $(1,0)$ and $(0,1)$. We claim this mechanism is the same as $(1,1)-\mathrm{C} 1$ Mechanism. The proof is rather easy. Assume there exists a group of $W_{0}=f\left(A_{0}, B_{0}\right)$ that $x_{W} \neq \max \left\{x_{A}, x_{B}\right\}$. Obviously $y_{A} \neq y_{B}, x_{A} \neq x_{B}$, and $W_{0}, A_{0}, B_{0}$ cannot locate at the same line, otherwise it will contradict the condition at once. Because $\angle A_{0} W_{0} B_{0}=90^{\circ}$, then if we move two lines $y=0$ and $x=0$, we will finally find that one of the two lines will have 2 intersections with broken line $A_{0} W_{0} B_{0}$ and let the two intersections on $\overline{A_{0} W_{0}}$ and $\overline{W_{0} B_{0}}$ be $C$ and $D$ (if there are infinite intersections, we can let one of the two points be $W_{0}$ and the other be $A_{0}$ or $B_{0}$, then there must be one contradiction in these two situations. In other 3 cases, this discussion will be omitted due to the length), thus we have $f(C, D)=W_{0}$, which contradicts with the conditions. In the same way we can also prove $y_{W} \neq \max \left\{y_{A}, y_{B}\right\}$ will lead to a contradiction, too. Therefore, this is the same as C1 Mechanism. In summary, the four situations are the 4 combinations of C1 Mechanism with different parameters.

(2) $f((0,-1),(0,1))=(0, \pm 1)$ and $f((-1,0),(1,0))=\left(\cos \theta_{1}, \sin \theta_{1}\right)$, where $\theta_{1} \neq 0, \pi$ (same in the following (4)). Out of symmetry, we only consider $f((0,-1),(0,1))=(0, \pm 1)$ and $\theta_{1} \in(0, \pi)$ (If $\theta_{1} \in(\pi, 2 \pi)$ then we can use the same method below to lead to a contradiction). According to Lemma 2, for $\forall C \in \overline{A_{0} W_{0}}, \forall D \in \overline{B_{0} W_{0}}$, we have $f(C, D)=W_{0}$, which is the same as case 2 in C2 Mechanism when $\left(y_{A}-y_{B}\right) /\left(x_{A}-x_{B}\right) \in\left[\sin \theta_{1} /\left(\cos \theta_{1}-1\right), \sin \theta_{1} /\left(1+\cos \theta_{1}\right)\right]$. When $\left(y_{A}-y_{B}\right) /\left(x_{A}-x_{B}\right)>\sin \theta_{1} /\left(1+\cos \theta_{1}\right)$ or $<\sin \theta_{1} /\left(\cos \theta_{1}-1\right)$, we can use the same method as (1) to prove $W$ locates at agents with larger $y$ axis, which is the same as $\mathrm{C} 2$ Mechanism. In summary, this case means $\left(\frac{\sin \theta_{1}}{1+\cos \theta_{1}}\right)-\mathrm{C} 2$ Mechanism. 
(3) $f((0,-1),(0,1))=\left(\cos \theta_{2}, \sin \theta_{2}\right)$ and $f((-1,0),(1,0))=( \pm 1,0)$, where $\theta_{2} \neq 0.5 \pi, 1.5 \pi$ (same in the following $\left.(4)\right)$. This case is the same as $\left(\frac{\cos \theta_{2}}{1+\sin \theta_{2}}\right)-\mathrm{C} 3$ Mechanism. The proof is similar to (2), thus omitted.

(4) $f((0,-1),(0,1))=\left(\cos \theta_{2}, \sin \theta_{2}\right)$ and $f((-1,0),(1,0))=\left(\cos \theta_{1}, \sin \theta_{1}\right)$. In fact, this case cannot exist. Let $A_{0}(-1,0), B_{0}(1,0), A_{1}(0,-1), B_{1}(0,1)$. We can draw a line which can be moved in the space and let it has 2 intersections with broken lines $A_{0} W_{0} B_{0}$ and $A_{1} W_{1} B_{1}$ and the intersections are $C_{0}, D_{0}$ and $C_{1}, D_{1}$ respectively. Considering $f\left(C_{0}, D_{0}\right)=W_{0}$ and $f\left(C_{1}, D_{1}\right)=W_{1}$, we find that considering translational-invariance and scalability, the contradiction is obvious.

\section{Discussion}

In this section we will discuss the general median mechanism and the lower bound of the maximum cost view.

Mechanism 4 : (General Median Mechanism). Given location of $n$ agents, let $W$ be the output, then in every dimension, $W$ 's coordinate is equal to agents' median coordinate in this dimension. If there are 2 median coordinates, then we select the larger one.

In fact, when there are 2 median coordinates, it does not matter if we select the larger one or the smaller one.

Lemma 6. The General Median Mechanism in Euclidean space fits Conjecture 1 if and only if dimension $m \leq 2$.

Proof. Obviously this mechanism is unanimous, translation-invariant, scalable, and much literature have proved that it's strategyproof in $L_{p}$ space. When $m=$ 1 , Conjecture 1 is also obvious.

When $m=2$, Let's recall Theorem 2. If $\exists i \in N, W=A_{i}$, then for any $j \in N$, we have $\overrightarrow{W A_{i}} \cdot \overrightarrow{W A_{j}}=0$. If for $\forall i \in N, W \neq A_{i}$, then assuming $W(0, \ldots, 0)$, there must exists $A_{s}, A_{t}$ that they locate on different axes, meaning $\overrightarrow{W A_{s}} \cdot \overrightarrow{W A_{t}}=0$.

When $m \geq 3$, we can give an counter-example with 3 agents. Let them be $A_{1}(0,1,-1,0, \ldots, 0), A_{2}(-1,0,1,0, \ldots, 0)$ and $A_{3}(1,-1,0,0, \ldots, 0)$. Then the output is $(0,0, \ldots, 0)$. But this is contradict with Conjecture 1 .

In the end, we use our tool to prove a lower bound of 2 in the maximum cost view.

Lemma 7. In any $L_{p}$ space and maximum cost view, the lower bound of deterministic strategyproof mechanism is 2 .

Proof. Assume $f(A, B)=W$ and $A, B$ are the only two agents, then according to the tool for the proof of Theorem 5 , we have $f(W, B)=B$, which means that if there two agents located at $W$ and $B$, then the maximum cost of $f$ is always at least twice the optimal maximum $\operatorname{cost} d(W, B) / 2$.

Many works proves this result in one-dimensional space and we give a simple proof of the multi-dimensional space. 


\section{References}

1. Alon, N., Feldman, M., Procaccia, A.D., Tennenholtz, M.: Strategyproof approximation of the minimax on networks. Mathematics of Operations Research 35(3), 513-526 (2010)

2. Barberà, S., Gul, F., Stacchetti, E.: Generalized median voter schemes and committees. Journal of Economic Theory 61(2), 262-289 (1993)

3. Barberà, S., Massó, J., Serizawa, S.: Strategy-proof voting on compact ranges. games and economic behavior 25(2), 272-291 (1998)

4. Border, K.C., Jordan, J.S.: Straightforward elections, unanimity and phantom voters. The Review of Economic Studies 50(1), 153-170 (1983)

5. Feigenbaum, I., Sethuraman, J., Ye, C.: Approximately optimal mechanisms for strategyproof facility location: Minimizing lp norm of costs. Mathematics of Operations Research 42(2), 434-447 (2017)

6. Filos-Ratsikas, A., Li, M., Zhang, J., Zhang, Q.: Facility location with doublepeaked preferences. Autonomous Agents and Multi-Agent Systems 31(6), 12091235 (2017)

7. Fong, K.C., Li, M., Lu, P., Todo, T., Yokoo, M.: Facility location games with fractional preferences. In: 32nd AAAI Conference on Artificial Intelligence, AAAI 2018. pp. 1039-1046. AAAI Press (2018)

8. Fotakis, D., Tzamos, C.: Winner-imposing strategyproof mechanisms for multiple facility location games. Theoretical Computer Science 472, 90-103 (2013)

9. Li, M., Lu, P., Yao, Y., Zhang, J.: Strategyproof mechanism for two heterogeneous facilities with constant approximation ratio. arXiv preprint arXiv:1907.08918 (2019)

10. Moulin, H.: On strategy-proofness and single peakedness. Public Choice 35(4), 437-455 (1980)

11. Procaccia, A.D., Tennenholtz, M.: Approximate mechanism design without money. In: Proceedings of the 10th ACM conference on Electronic commerce. pp. 177-186 (2009)

12. Serafino, P., Ventre, C.: Heterogeneous facility location without money on the line. In: ECAI. pp. 807-812 (2014)

13. Tang, P., Yu, D., Zhao, S.: Characterization of group-strategyproof mechanisms for facility location in strictly convex space. In: Proceedings of the 21st ACM Conference on Economics and Computation. pp. 133-157 (2020)

14. Yuan, H., Wang, K., Fong, K.C., Zhang, Y., Li, M.: Facility location games with optional preference. In: Proceedings of the Twenty-second European Conference on Artificial Intelligence. pp. 1520-1527 (2016) 